\title{
O PROCESSO DE ENSINO-APRENDIZAGEM POR MEIO DE SIMULAÇÃO E JOGO:
}

\author{
UMA ANÁLISE BIBLIOMÉTRICA
}

\author{
Simone Sartori ${ }^{1}$ \\ Marcelo Merizio ${ }^{2}$ \\ Sidnei Gripa ${ }^{3}$
}

Recebido em: 31 dez. 2019

Aceito em: 7 fev. 2020

Como citar este artigo: SARTORI, S.; MERIZIO, M.; GRIPA, S. O PROCESSO DE ENSINO-

APRENDIZAGEM POR MEIO DE SIMULAÇÃO E JOGO: UMA ANÁLISE BIBLIOMÉTRICA. Revista

Visão: Gestão Organizacional, v.9, n.1, p.84-99, 2020. DOI:

https://doi.org/10.33362/visao.v9i1.2166

Resumo: O processo de ensino-aprendizagem com efetiva participação dos alunos vem sendo discutido nos últimos anos. Entretanto, a contribuição deste processo e as formas ainda são incipientes. O objetivo deste estudo foi analisar a contribuição dos modelos ou formas de ensino-aprendizagem na área de engenharia de produção e áreas próximas. Foram selecionados artigos científicos, de acordo com o interesse dos pesquisadores. A base Science Direct foi usada, considerando o período de 2009 a 2019 e foram selecionados 31 artigos para compor o portfólio bibliográfico (PB). Utilizou-se sete marcadores bibliométricos para realizar a análise: periódicos e fator de impacto, as citações por artigo, palavras-chave, autores, instituição, país e rede bibliométrica. Os periódicos mais citados no PB: Destacam-se "Computers \& Education" e "The International Journal of Management"; a origem dos autores com maior participação é do Reino Unido e da University of Turku. Os termos principais encontrados nos resumos do PB foram simulation game, challenge, education, process, development, student, active learning, team, gaming session, player, entre outros elementares. Já análise de conteúdo permitiu demonstrar a importância da simulação no processo de ensinoaprendizagem e uso de softwares/estratégias neste processo.

Palavras-Chave: Simulação. Processo de Ensino-aprendizagem. Engenharia de Produção.

TEACHING-LEARNING PROCESS WITH SIMULATIONS AND GAME: A BIBLIOMETRIC ANALYSIS

Abstract: The teaching-learning process with effective student participation has been discussed in recent years. However, the contribution of this process and the forms are still incipient. The aim of this study was to analyze the contribution of models or forms of teaching-learning in the area of production engineering and áreas close. Scientific articles were selected, according to the researchers' interest. A reference survey was made in

\footnotetext{
${ }^{1}$ Centro Universitário de Brusque - UNIFEBE. E-mail: simone.sartori@unifebe.edu.br.

${ }^{2}$ Centro Universitário de Brusque - UNIFEBE. E-mail: mmerizio@unifebe.edu.br.

${ }^{3}$ Centro Universitário de Brusque - UNIFEBE. E-mail: gripa@unifebe.edu.br.
} 
the database Science Direct, considering the period from 2009 to 2019 and were selected 31 articles to compose the bibliographic portfolio (BP). We used seven bibliometric markers when performing these analyses: journals and impact factor, citations per article, keywords, authors, institution, country and bibliometric networks. The most cited journals in BP: "Computers \& Education" and "The International Journal of Management"; the origin of the authors with the largest participation is from the United Kingdom and the University of Turku. The main issues found in the BP were simulation game, challenge, education, process, development, student, active learning, team, gaming session, player and others. In the content analysis was demonstrated the importance of simulation in the teaching-learning process and the use of software / strategies in this process.

Keywords: Simulation. The teaching-learning process. Industrial Engineering.

\section{INTRODUÇÃO}

Nas últimas décadas, os sistemas de produção tornaram-se complexos para atender aos requisitos da crescente demanda de qualidade e flexibilidade da produção (POPOVICS; MONOSTORI, 2016), além de produzir com alta eficiência (LACHENMAIER; LASI; KEMPER, 2017). Entretanto, muitos problemas estão na capacidade de reconhecer desperdícios e tomar medidas para melhorar o sistema de produção (WYRWICKA; MRUGALSKA, 2017).

Cursos tradicionais, como de Administração e Engenharia de Produção, possuem conteúdos curriculares voltados à gestão da produção. No modelo tradicional de ensino, em que o professor é o agente indutor do conhecimento (GIMENEZ-LOPEZ et al., 2010), fazia sentido quando o acesso à informação era difícil (MORAN, 2015). Essas metodologias predominantes no ensino são as dedutivas: o professor transmite primeiro a teoria e depois o aluno deve aplicá-la a situações mais específicas.

Esta aprendizagem ocorre por meio da transmissão é importante, mas a aprendizagem por questionamento e experimentação é mais relevante para uma compreensão mais ampla e profunda (BACICH; MORAN, 2017). Bem como, com os avanços dos sistemas de informação e comunicação, é possível aprender em qualquer lugar, a qualquer hora e com muitas pessoas diferentes (MORAN, 2015).

Nos últimos anos, há ênfase em combinar metodologias participativas - também chamada de metodologias ativas - em que unem as vantagens das metodologias indutivas e das metodologias dedutivas. Nas metodologias participativas usam-se técnicas que exigem participação dinâmica do aluno nas atividades de aprendizagem, em que os alunos não são receptores passivos, mas sujeitos ativos no processo de aprendizagem (GIMENEZ-LOPEZ et al., 2010).

Portanto, neste contexto, faz-se necessário o uso de um conjunto de processos, procedimentos, técnicas e ferramentas que envolvem o aluno ativamente no processo de 
ensino (MOYA, 2017), que podem ser adaptadas entre o individual e o coletivo (BACICH; MORAN, 2017). Pode-se começar por projetos, pesquisa, leituras prévias e produções dos alunos e depois promover aprofundamentos em classe com a orientação do professor (FONSECA; GOMES, 2013).

Além disso, o uso dos exemplos do mundo real, relacionados ao conteúdo do assunto, dá um incentivo maior aos alunos dada a aplicação prática (GIMENEZ-LOPEZ et al., 2010). Em todos os casos, faz-se necessário uma combinação de métodos de aprendizagem inovadores, uma vez que o novo papel do professor no processo de ensino-aprendizagem deve ser o de ajudar o aluno no processo de aquisição de competências (MOYA, 2017).

Nesse sentido, para atingir esse objetivo de maneira eficiente, a universidade deve propor a transição de um modelo educacional centrado no ensino para um modelo centrado na aprendizagem. O objetivo deste artigo é identificar as práticas que estão sendo utilizadas por meio do uso de simulações - simulação física e computacional - ou jogos visando aumentar o conhecimento dos alunos para atuar no mercado de trabalho de forma mais efetiva.

\section{MATERIAIS E MÉTODOS}

A construção do conhecimento acerca de um contexto de interesse do pesquisador deve basear-se na revisão da literatura acerca do tema (AFONSO et al., 2011), de acordo com o interesse e as delimitações do pesquisador (ENSSLIN, 2012). Foram definidos três eixos de pesquisa: (i) o primeiro eixo corresponde ao tema central do trabalho - Gestão da Produção; (ii) o segundo eixo está relacionado a aplicação do primeiro eixo, ou seja, Processo de Ensino e Aprendizagem; e, (iii) o terceiro corresponde à Simulação, pois objetiva subsidiar os processos decisórios na busca de aperfeiçoamentos na gestão da produção. A pesquisa compreendeu as seguintes etapas: (i) definição das palavras-chave, (ii) seleção da base de dados iii) coleta e análise; e, iv) seleção do Portfólio Bibliográfico (PB).

As principais palavras-chave utilizadas neste estudo foram escolhidas com atenção ao objetivo principal deste estudo, que é explorar a relação entre o processo de aprendizagem e o uso de simulação.

A busca realizada no mês de maio de 2019, na base Science Direct, com as seguintes palavras-chave: "Production management" / "industrial production" and "Teaching strategies"; "Production management" / "industrial production" and "learning process"; "Production management" / "industrial production" and simulation; "Production management" / learning and "production management"; "industrial production" and "simulation games"; Learning and "simulation games"; simulation and learning; Gamification and "Production management" / "industrial production"; "Production management" / "industrial production" and "Game education"; production games; "simulation game". Foram considerados os campos de busca: 
Title, abstract or author-specified Keywords. A pesquisa retornou 1.308 artigos brutos.

Após a análise individual de cada artigo, foram selecionados 31 artigos para compor o Portfólio Bibliográfico (PB) relevante, alinhado ao tema do pesquisador e o objetivo da pesquisa. Por conseguinte, buscou-se apresentar a contribuição de cada artigo. Por fim, usouse do software VOSviewer com o objetivo de visualização das redes de ocorrência dos termos extraídos dos resumos.

\section{RESULTADOS E DISCUSSÕES}

Nos últimos anos, houve um crescimento significativo de pesquisas acerca de metodologias de ensino inovadoras voltadas para a engenharia. Os artigos científicos são compilados e indexados em grandes bancos de dados, que permitem medir diferentes aspectos, como: autores, palavras-chave, citações, colaboração institucional, contribuição da pesquisa.

A primeira análise compreende o uso de palavras-chave, conforme apresentado na Figura 1. Destacam-se Simulation games, Simulations, Adult learning, Business simulation games, Digital games, Game-based learning, Interactive learning environments, Simulation e Teaching.

Figura 1 - Palavras-chave da pesquisa

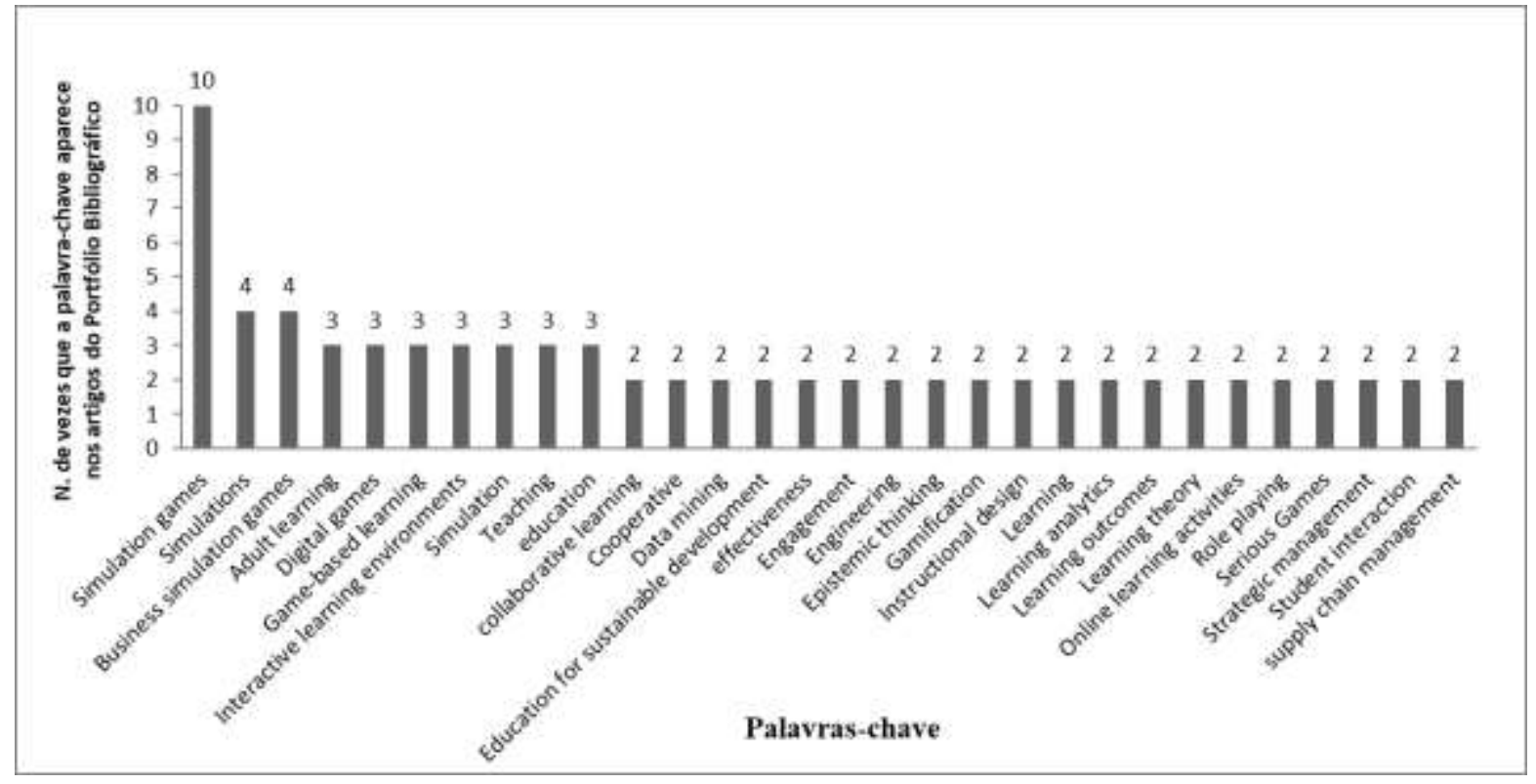

Fonte: Os autores (2019).

Por conseguinte, foi avaliado os periódicos científicos do PB, considerando a sua relevância (Figura 2). O JCR apresenta as conexões criadas pela comunidade de pesquisa por meio da entrega de uma variedade de dados, métricas e análises independentes do editor, dos 
periódicos mais impactantes do mundo. Portanto, reflete o número médio de citações de artigos científicos publicados em determinado periódico (WEB OF KNOWLEDGE, 2012). Destacam-se Journal of Clener Production (6,395), Computers \& Education $(5,627)$ e Information Sciences $(5,524)$. Considerando o tema da pesquisa, todos os periódicos agregam na formação e no compartilhamento do conhecimento.

Figura 2 - Relevância do assunto para o Portfólio Bibliográfico (JCR)

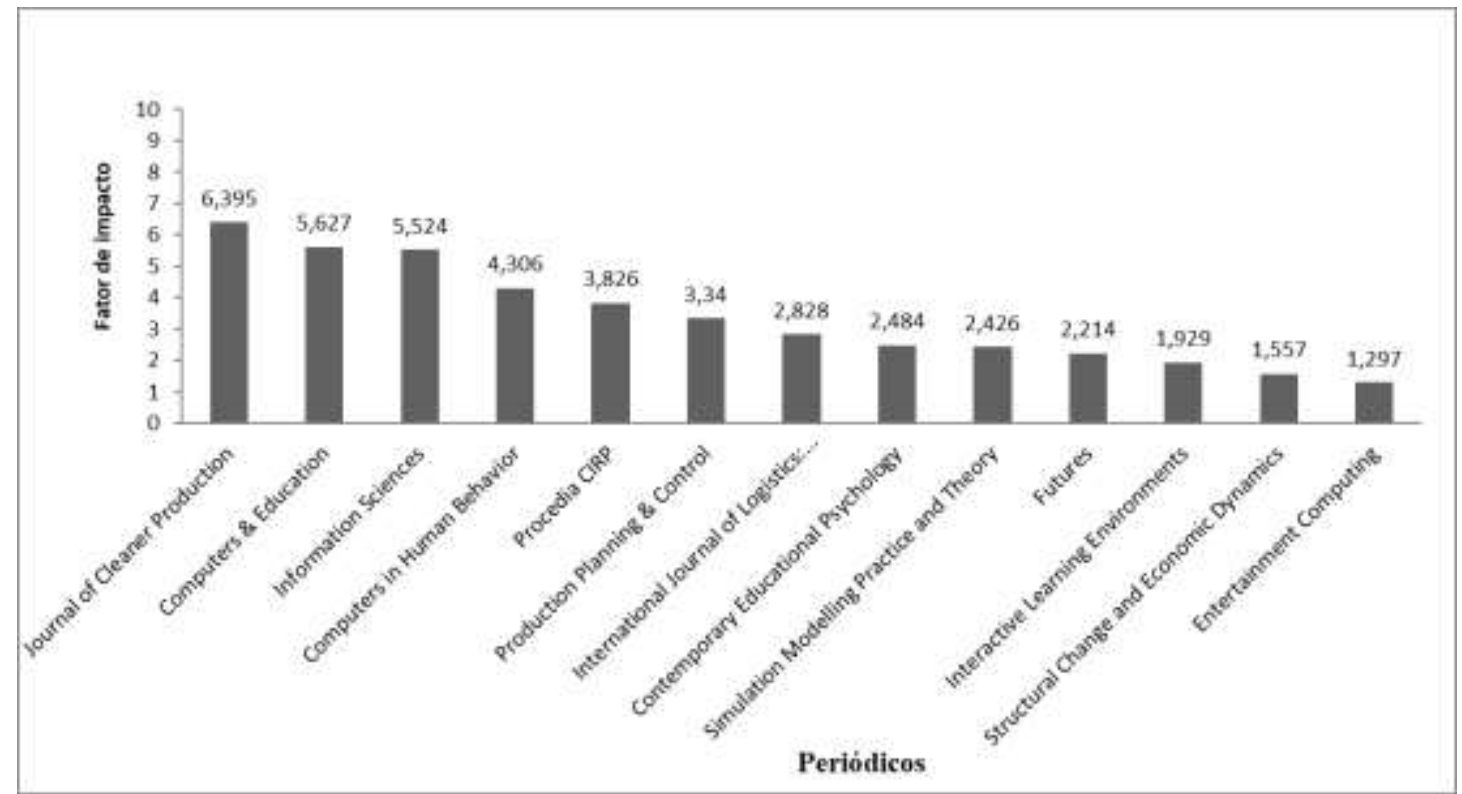

Fonte: Os autores (2019).

A terceira análise evidenciou os autores do PB que se destacam nas publicações, conforme evidenciado na Figura 3. O PB está composto por 83 autores. Destes, o autor Timo Lainema está presente em três artigos. Os demais autores apresentados possuem duas publicações ou apenas uma. 
Figura 3-Autores do PB.

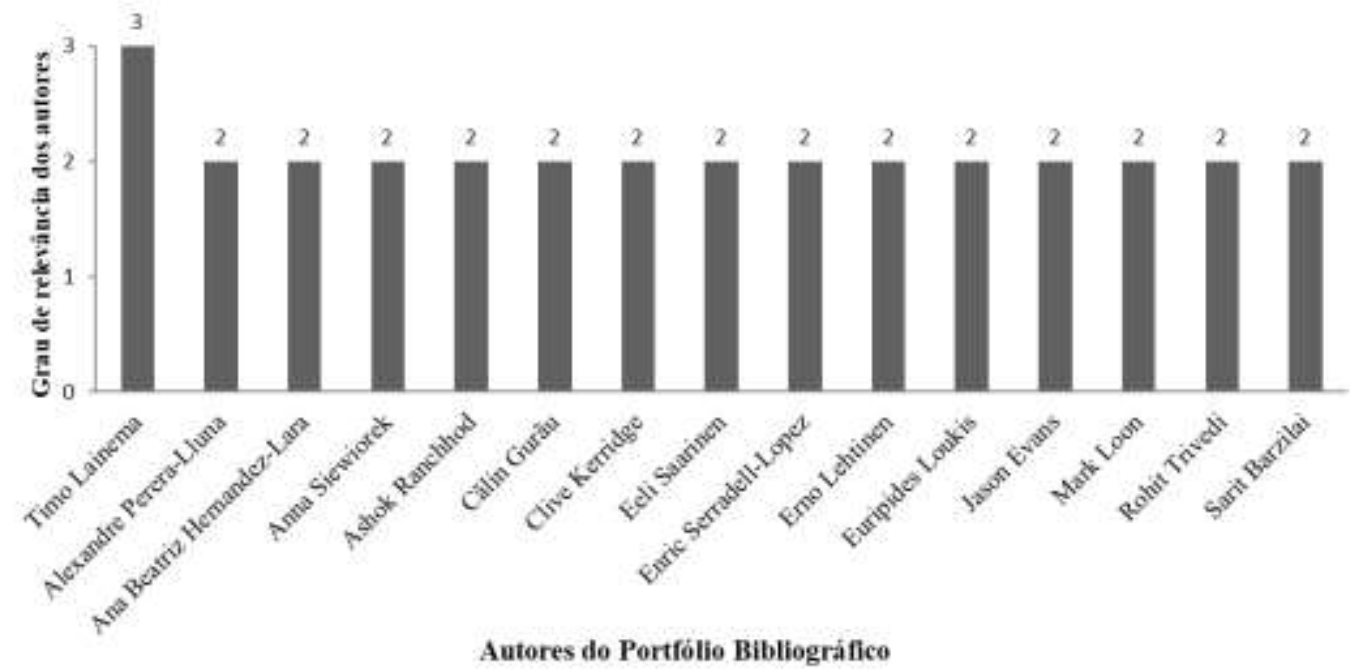

Fonte: Os autores

A Figura 4 apresenta os periódicos que mais se repetem no PB. Destacam-se "Computers \& Education" e "The International Journal of Management". Os periódicos mais citados refletem a importância do assunto na área. Pesquisadores, professores e instituições podem obter informações importantes sobre o impacto individual ou agregado, além de estratégias de pesquisa.

Figura 4 - Periódicos de relevância no PB.

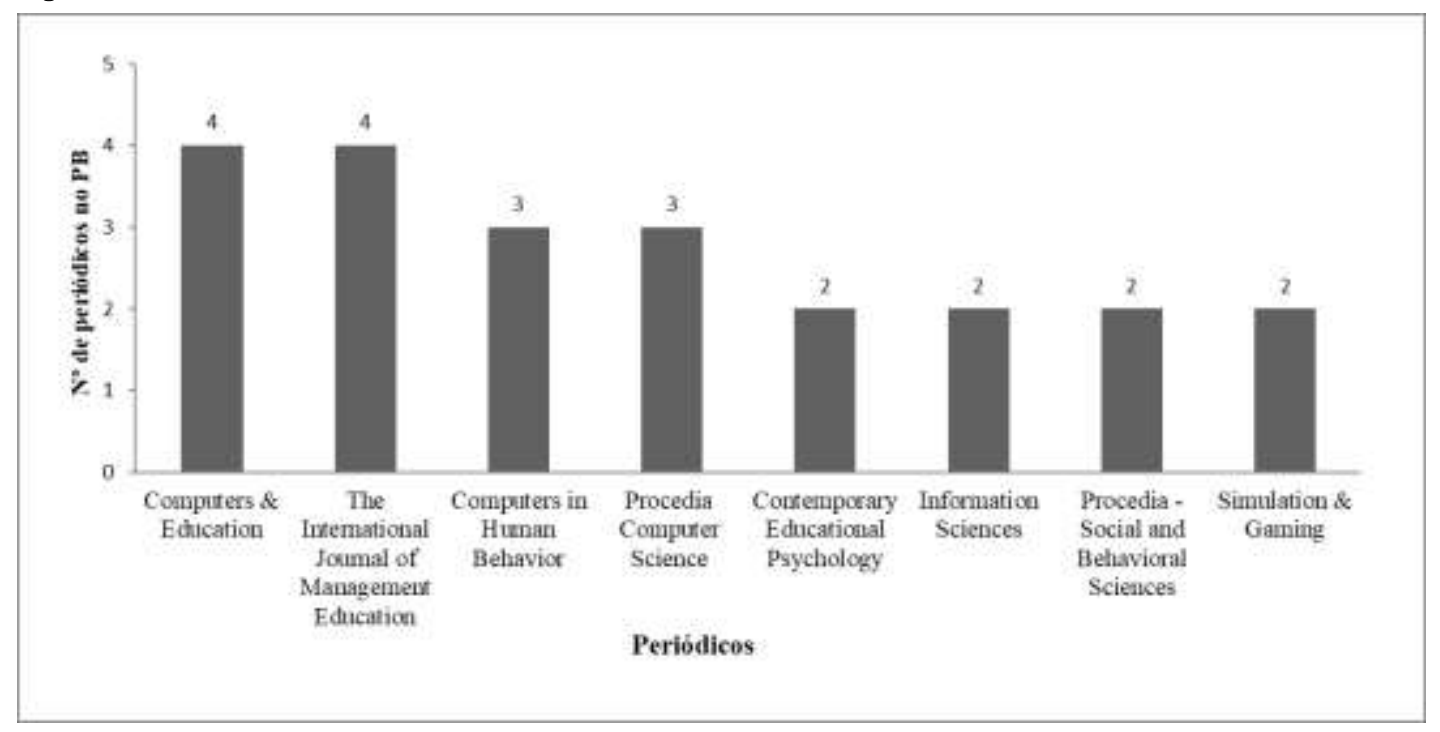

Fonte: os autores (2019).

A Figura 5 apresenta a publicação científica de acordo com origem institucional e país. Os países Reino Unido, Espanha, Alemanha e Finlândia possui a maior participação no PB. Já as 
instituições que mais publicaram no PB foram University of Turku e Universitat Autònoma de Barcelona.

Figura 5 - Países e instituições no PB.

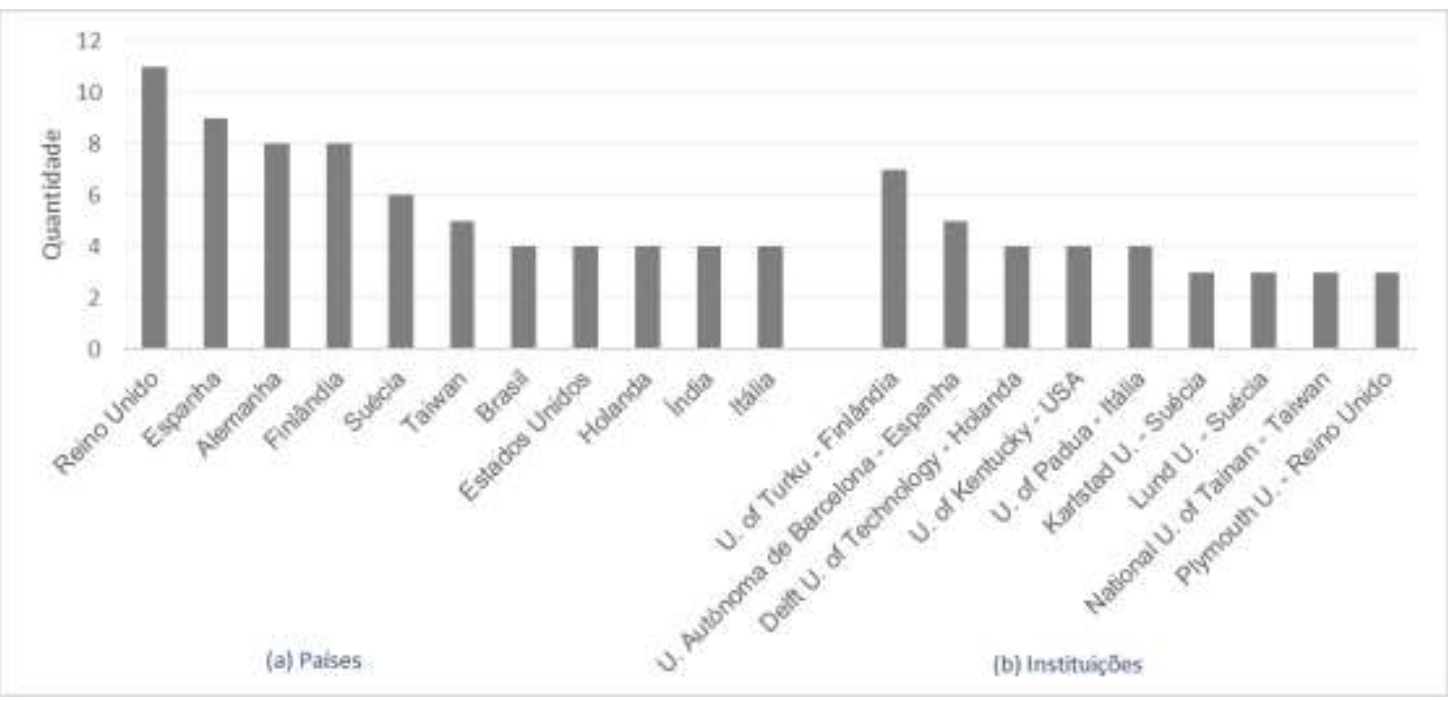

Fonte: os autores (2019).

No entanto, a importância dos estudos bibliométricos vai além do nível institucional. Eles podem ajudar novos pesquisadores de uma disciplina a entender a extensão de um tópico, tendências emergentes e sua evolução ao longo do tempo (MEREDIZ-SOLÀ; BARIVIERA, 2019). Sendo diferente de um levantamento bibliográfico tradicional, o Quadro 1 apresenta os artigos do PB com ênfase para os meios e os principais resultados encontrados.

Quadro 1 - Análise do PB

\begin{tabular}{|c|c|c|c|}
\hline \multicolumn{2}{|c|}{ Dinâmica da pesquisa } & Resultados & Autores \\
\hline 1 & $\begin{array}{l}\text { - Desktop games; } \\
\text { - Karlstad Lean } \\
\text { Factory (KLF); } \\
\text { - Estudantes e } \\
\text { colaboradores indústria }\end{array}$ & $\begin{array}{l}\text { - Os atuais métodos de simulação em lean são subjetivos } \\
\text { e não uniformes; } \\
\text { - Para os estudantes e colaboradores industriais, efeitos } \\
\text { de treinamento e imersão tendem a ser maiores ao usar } \\
\text { simuladores em escala real. }\end{array}$ & $\begin{array}{l}\text { Vin, Jacobsson } \\
\text { e Odhe (2018) }\end{array}$ \\
\hline 2 & $\begin{array}{l}\text { - Markstrat simulation } \\
\text { game } \\
\text { - } 305 \text { estudantes; } \\
\text { - Aprendizagem } \\
\text { experimental. }\end{array}$ & $\begin{array}{l}\text { - A simulação permite melhor entendimento conceitual e } \\
\text { desenvolvimento de habilidades profissionais; } \\
\text { - O modelo apresentado no estudo é generalizável para } \\
\text { outros jogos de simulação e para outras disciplinas } \\
\text { acadêmicas que implementam a mesma abordagem de } \\
\text { aprendizado experimental. }\end{array}$ & $\begin{array}{l}\text { Ranchhod et } \\
\text { al. (2014) }\end{array}$ \\
\hline 3 & $\begin{array}{l}\text { - Simulação; } \\
\text { - Avaliação baseada no } \\
\text { erro na primeira e } \\
\text { segunda rodada; } \\
\text { - } 100 \text { equipes. }\end{array}$ & $\begin{array}{l}\text { - Embora as habilidades de tomada de decisão possam } \\
\text { ser adquiridas com métodos de ensino tradicionais, os } \\
\text { jogos de simulação são mais eficazes quando os alunos } \\
\text { precisam desenvolver habilidades de tomada de decisão } \\
\text { para gerenciar situações complexas e dinâmicas. }\end{array}$ & $\begin{array}{l}\text { Pasin e Giroux } \\
\text { (2011). }\end{array}$ \\
\hline 4 & $\begin{array}{l}\text { - Simulação virtual; } \\
\text { - Equipes virtuais e alguns }\end{array}$ & $\begin{array}{l}\text { - Os jogos de simulação têm o potencial de ser } \\
\text { usado no ensino superior para exercitar as habilidades }\end{array}$ & $\begin{array}{l}\text { Siewiorek et } \\
\text { al. (2012) }\end{array}$ \\
\hline
\end{tabular}




\begin{tabular}{|c|c|c|c|}
\hline & $\begin{array}{l}\text { membros satélites; } \\
\text { - } 41 \text { estudantes de pós- } \\
\text { graduação. }\end{array}$ & $\begin{array}{l}\text { de liderança } \\
\text { - Considerando o tipo de papel do aluno na equipe, } \\
\text { algumas características de liderança surgiram } \\
\text { espontaneamente. }\end{array}$ & \\
\hline 5 & $\begin{array}{l}\text { - Estratégias de } \\
\text { aprendizado: prática, } \\
\text { feeedback,questionário, } \\
\text { aprendizagem } \\
\text { coopeerativa, multimídia } \\
\text { interativa, etc.; } \\
\text { - } 126 \text { artigos revisados. }\end{array}$ & $\begin{array}{l}\text { - São três as estratégias mais promissoras para alavancar } \\
\text { o aprendizado em escala, como: aprendizado } \\
\text { cooperativo, simulações/ jogos e multimídia interativa. }\end{array}$ & $\begin{array}{l}\text { Davis et al. } \\
\text { (2018) }\end{array}$ \\
\hline 6 & $\begin{array}{l}\text { - Simulação e jogo digital; } \\
\text { - Análise de correlação de } \\
\text { elementos; } \\
\text { - } 50 \text { estudantes jogadores } \\
\text { de SimCity. }\end{array}$ & $\begin{array}{l}\text { - Houve muitos benefícios a partir da simulação: } \\
\text { desenvolvimento da capacidade de solução de } \\
\text { problemas, criatividade, desenvolvimento do senso de } \\
\text { responsabilidade, aumento da experiência de } \\
\text { planejamento e organizacional, fortalecimento da } \\
\text { tomada de decisão e liderança, melhoria da capacidade } \\
\text { de controle e auto realização; } \\
\text { - A simulação é útil no processo de ensino e } \\
\text { aprendizagem, tornando-se uma estratégia que vai além } \\
\text { da sala de aula tradicional. }\end{array}$ & $\begin{array}{l}\text { Lim e Lim } \\
(2014)\end{array}$ \\
\hline 7 & $\begin{array}{l}\text { - Jogo de simulação } \\
\text { RealGame; } \\
\text { - Formação de grupos e } \\
\text { rodadas de jogo; } \\
\text { - } 129 \text { estudantes. }\end{array}$ & $\begin{array}{l}\text { - A estrutura do jogo pode ser usada para analisar a } \\
\text { qualidade da experiência do jogo, mas não fornece } \\
\text { informações detalhadas sobre os desafios (gaps) ou os } \\
\text { destaques do jogo, ou seja, a escala de experiência do } \\
\text { jogo. }\end{array}$ & $\begin{array}{l}\text { Kiili et al. } \\
(2014)\end{array}$ \\
\hline 8 & $\begin{array}{l}\text { - Jogo de simulação; } \\
\text { - Testes de múltipla } \\
\text { escolha; } \\
\text { - } 25 \text { estudantes de } \\
\text { mestrado em engenharia. }\end{array}$ & $\begin{array}{l}\text { - Alto nível de envolvimento no jogo e entre os alunos } \\
\text { participante; } \\
\text { - O conjunto de dados não foi útil para avaliar } \\
\text { estatisticamente o impacto da gamificação, muitos } \\
\text { estudantes afirmaram que o design do teste gamificado } \\
\text { pressionou-os e os ajudou a relaxar; } \\
\text { - Quase 50\% dos estudantes preferem testes de múltipla } \\
\text { escolha, pois há aprendizado orientado para o teste e, } \\
\text { portanto, melhores nota; } \\
\text { - Ao planejar testes gamificados, o tempo precisa ser } \\
\text { levado em consideração. }\end{array}$ & $\begin{array}{l}\text { Severengiz et } \\
\text { al. (2018) }\end{array}$ \\
\hline 9 & $\begin{array}{l}\text { - Conceitual e descritivo; } \\
\text { - Proposta de um } \\
\text { framework para ensino- } \\
\text { aprendizagem. }\end{array}$ & $\begin{array}{l}\text { - O estudo demonstra a importância da TI como suporte } \\
\text { aos conceitos, ferramentas e práticas para o ensino e } \\
\text { aprendizagem de qualidade, voltando-se para a Gestão } \\
\text { do Conhecimento. }\end{array}$ & $\begin{array}{l}\text { Bhusry e } \\
\text { Ranjan (2011) }\end{array}$ \\
\hline 10 & $\begin{array}{l}\text { - Business Strategy Game } \\
\text { (BSG); } \\
\text { - } 27 \text { estudantes; } \\
\text { - Pesquisa-ação baseada } \\
\text { em incidentes. }\end{array}$ & $\begin{array}{l}\text { - A capacidade dos alunos para compactar prazos e } \\
\text { gerenciar incidentes permite que eles aprendam de uma } \\
\text { maneira que não seria possível no mundo real, } \\
\text { consequentemente, o jogo potencializa o aumento da } \\
\text { aprendizagem. }\end{array}$ & $\begin{array}{l}\text { Lean, Moizer e } \\
\text { Newbery } \\
\text { (2014) }\end{array}$ \\
\hline 11 & $\begin{array}{l}\text { - Aprendizagem baseada } \\
\text { em (PBL); } \\
\text { - Análise de literatura }\end{array}$ & $\begin{array}{l}\text { - Quatro lacunas foram encontradas em } \\
\text { projetos de simulação existentes: falta de ênfase nas } \\
\text { habilidades sociais, foco incorreto em }\end{array}$ & $\begin{array}{l}\text { Badurdeen et } \\
\text { al. (2010) }\end{array}$ \\
\hline
\end{tabular}




\begin{tabular}{|c|c|c|c|}
\hline & $\begin{array}{l}\text { sobre as simulações } \\
\text { existentes; }\end{array}$ & $\begin{array}{l}\text { lean, incompreensão do papel principal do facilitador e } \\
\text { falta de realismo; } \\
\text { - São sugeridas instruções para estudo e melhoria no } \\
\text { projeto de simulação lean. }\end{array}$ & \\
\hline 12 & $\begin{array}{l}\text { - Simulação; } \\
\text { - LEGO }{ }^{\circledR} \text { Serious Play; } \\
\text { - Percepção antes e depois } \\
\text { da simulação; } \\
\text { - } 47 \text { estudantes. }\end{array}$ & $\begin{array}{l}\text { - O método LEGO Serious Play os ajudou a lidar melhor } \\
\text { com os desafios do trabalho em equipe, influências das } \\
\text { partes interessadas, fatores de risco e projetos } \\
\text { imprevisíveis; } \\
\text { - O jogo de simulação de negócios é eficaz para o } \\
\text { aprendizado e treinamento, desenvolvimento } \\
\text { habilidades relevantes necessárias para os gerentes de } \\
\text { projeto. }\end{array}$ & $\begin{array}{l}\text { Geithner e } \\
\text { Menzel (2014) }\end{array}$ \\
\hline 13 & $\begin{array}{l}\text { - Simulação; } \\
\text { - Jogo digital; } \\
\text { - Survey. }\end{array}$ & $\begin{array}{l}\text { - O jogo melhorou o crescimento cognitivo dos } \\
\text { jogadores, bem como o ambiente simulado criado pelo } \\
\text { jogo mesclou realidade e virtualidade com sucesso. }\end{array}$ & $\begin{array}{l}\text { Shih, Jheng e } \\
\text { Tseng (2015) }\end{array}$ \\
\hline 14 & $\begin{array}{l}\text { - Múltiplos casos; } \\
\text { - Simulação; } \\
\text { - EnerCities; } \\
\text { - } 14 \text { estudantes com } \\
\text { média de 14,7 anos de } \\
\text { idade. }\end{array}$ & $\begin{array}{l}\text { - Os jogadores adotaram objetivos epistêmicos } \\
\text { específicos, mas embora fossem hábeis em atingir } \\
\text { objetivos relacionados ao conhecimento no jogo e } \\
\text { sabendo sobre o jogo, eles não se engajaram na } \\
\text { representação do jogo; } \\
\text { - O estudo lança luz sobre como os jogos podem apoiar o } \\
\text { pensamento epistêmico. }\end{array}$ & Barzilai (2017) \\
\hline 15 & $\begin{array}{l}\text { - Muitos cenários de } \\
\text { simulação com OPNET } \\
\text { Modeler; } \\
\text { - Poucos cenários } \\
\text { simulação com ferramenta } \\
\text { de gestão; } \\
\text { - Survey. }\end{array}$ & $\begin{array}{l}\text { - As mudanças na estratégia de ensino possibilitaram } \\
\text { constante melhoria em dois aspectos relacionados à } \\
\text { qualidade do ensino: i) notas acadêmicas dos alunos; e ii) } \\
\text { a percepção dos alunos sobre os conhecimentos e } \\
\text { habilidades adquiridos ao longo do módulo. }\end{array}$ & $\begin{array}{l}\text { Zarza et al. } \\
(2012)\end{array}$ \\
\hline 16 & $\begin{array}{l}\text { - Aprendizagem analítica e } \\
\text { técnica de data mining; } \\
\text { - Análise documental; } \\
\text { - Análise de discussão em } \\
\text { fóruns com } 362 \\
\text { estudantes de mestrado e } \\
\text { bacharel. }\end{array}$ & $\begin{array}{l}\text { - Os conteúdos mais frequentes nos fóruns de discussão } \\
\text { on-line dos alunos estavam relacionados, } \\
\text { primeiramente, aos parâmetros e recursos do jogo de } \\
\text { simulação de negócios e, em segundo lugar, a elementos } \\
\text { que promovam o processo de aprendizado dos alunos. } \\
\text { Os conteúdos com poder preditivo sobre os resultados } \\
\text { da aprendizagem estavam relacionados à incerteza, } \\
\text { tempo, interação, comunicação e colaboração, embora } \\
\text { nenhum desses elementos tenha influenciado a } \\
\text { avaliação do professor pela aprendizagem dos alunos. }\end{array}$ & $\begin{array}{l}\text { Hernandez- } \\
\text { Lara et al. } \\
(2019)\end{array}$ \\
\hline 17 & $\begin{array}{l}\text { - Simulação JA TITAN; } \\
\text { - Simulação MARKET } \\
\text { HERO; } \\
\text { - Survey. }\end{array}$ & $\begin{array}{l}\text { - O processo de ensino por meio da simulação é ativo e } \\
\text { fornece feedback importante entre as atividades dos } \\
\text { alunos e seus resultados; } \\
\text { - Os alunos destacam que a simulação é benéfica tanto } \\
\text { para estudos futuros quanto para ocupação futura, assim } \\
\text { como permite desenvolver habilidades comunicacionais, } \\
\text { motivação, tomada de decisão estratégica, usabilidade } \\
\text { do jogo para a prática, visão geral de uma organização, } \\
\text { entre outras vantagens. }\end{array}$ & $\begin{array}{l}\text { Peterková } \\
\text { Wozniaková } \\
(2015)\end{array}$ \\
\hline 18 & - Simulação LEGOLAS; & $\begin{array}{l}\text { - A simulação oferece uma oportunidade para os } \\
\text { participantes desenvolverem não apenas as suas }\end{array}$ & $\begin{array}{l}\text { Gabrielsson, } \\
\text { Tell e Politis }\end{array}$ \\
\hline
\end{tabular}




\begin{tabular}{|c|c|c|c|}
\hline & $\begin{array}{l}\text { - Equipes de estudantes; } \\
\text { - Estudo de caso. }\end{array}$ & $\begin{array}{l}\text { habilidades gerenciais, mas também uma cultura de } \\
\text { pesquisa como parte de sua vida profissional. } \\
\text { - Houve um alto envolvimento emocional dos } \\
\text { participantes e foi criada uma dinâmica durante a } \\
\text { simulação. }\end{array}$ & (2010) \\
\hline 19 & $\begin{array}{l}\text { - Simulação business } \\
\text { wargaming; } \\
\text { - Experiências do autor em } \\
\text { cursos de graduação e } \\
\text { pós-graduação. }\end{array}$ & $\begin{array}{l}\text { - As principais vantagens de um business wargame, por } \\
\text { meio da simulação, contribui para a dinâmica de ensino, } \\
\text { criatividade e discussões entre os participantes; } \\
\text { - Envolveu ativamente os participantes em uma } \\
\text { experiência de aprendizado orientada para o futuro em } \\
\text { ambientes dinâmicos e complexos. }\end{array}$ & $\begin{array}{l}\text { Schwarz } \\
(2013)\end{array}$ \\
\hline 20 & $\begin{array}{l}\text { - Simulação Napuro; } \\
\text { - Survey antes e após a } \\
\text { simulação. }\end{array}$ & $\begin{array}{l}\text { - A abordagem de simulação gera resultados de } \\
\text { aprendizado cognitivo e afetivo que, por sua vez, podem } \\
\text { afetar desenvolvimento de habilidades de pensamento } \\
\text { crítico pelos alunos. }\end{array}$ & $\begin{array}{l}\text { Gatti, Ulrich e } \\
\text { Seele (2019) }\end{array}$ \\
\hline 21 & $\begin{array}{l}\text { - Simulação SimGestion; } \\
\text { - Proposta de um modelo; } \\
\text { - Survey com } 360 \\
\text { estudantes de graduação. }\end{array}$ & $\begin{array}{l}\text { - Recomenda-se o jogo de simulação de negócios em } \\
\text { treinamento gerencial como ferramenta para promover } \\
\text { a motivação intrínseca entre os jogadores, promover o } \\
\text { engajamento, desenvolver habilidades e aumentar o } \\
\text { aprendizado no campo da administração; } \\
\text { - Tanto a motivação intrínseca quanto o engajamento } \\
\text { melhoram o desenvolvimento de habilidades genéricas } \\
\text { dos jogadores, como tomada de decisão, trabalho sob } \\
\text { pressão e trabalho em equipe. }\end{array}$ & $\begin{array}{l}\text { Buil, Catalán e } \\
\text { Martínez } \\
\text { (2019) }\end{array}$ \\
\hline 22 & $\begin{array}{l}\text { - Jogo de Simulação } \\
\text { Markstrat; } \\
\text { - } 305 \text { estudantes do UK; } \\
\text { - Equação estrutural. }\end{array}$ & $\begin{array}{l}\text { - Quatro tipos de valor educacional foram identificados: } \\
\text { geração de experiência, compreensão conceitual, } \\
\text { desenvolvimento de habilidades e avaliação afetiva; } \\
\text { - A geração de experiências tem um forte impacto no } \\
\text { entendimento conceitual e ambos têm impactos direto } \\
\text { no desenvolvimento de habilidades; } \\
\text { - O Markstrat é eficaz no desenvolvimento de } \\
\text { habilidades de tomada de decisão em situações } \\
\text { complexas e dinâmicas. }\end{array}$ & $\begin{array}{l}\text { Ranchhod et } \\
\text { al. (2014) }\end{array}$ \\
\hline 23 & $\begin{array}{l}\text { - Jogo de simulação } \\
\text { COSIGA e BEWARE; } \\
\text { - } 2 \text { estudos de caso com } \\
\text { estudantes de engenharia. }\end{array}$ & $\begin{array}{l}\text { - Os resultados do COSIGA (jogo de simulação no } \\
\text { desenvolvimento de produtos) cumpriram os objetivos } \\
\text { pedagógicos em projetar um novo produto de forma } \\
\text { multidisciplinar e colaborativa; } \\
\text { - O resultado da aplicação do BEWARE (jogo de } \\
\text { gerenciamento de riscos) possibilitou aumentar a } \\
\text { conscientização dos riscos nas redes de produção; } \\
\text { - Recomenda-se que os métodos de avaliação utilizados } \\
\text { regularmente durante o ensino precisam ser simples, } \\
\text { rápidos e fáceis de concluir; } \\
\text { - A inclusão de indicadores de desempenho permite que } \\
\text { os participantes e os facilitadores apontem a direção da } \\
\text { aprendizagem. }\end{array}$ & $\begin{array}{l}\text { Hauge e } \\
\text { Riedel (2012) }\end{array}$ \\
\hline 24 & $\begin{array}{l}\text { - Simulação RealGame; } \\
\text { - } 41 \text { estudantes de } \\
\text { graduação; } \\
\text { - Análise dos relatórios }\end{array}$ & $\begin{array}{l}\text { - Na simulação de negócios, características de liderança } \\
\text { surgiram espontaneamente; } \\
\text { - As experiências sobre liderança variaram entre as } \\
\text { equipes e dependiam do papel que os alunos tinham em }\end{array}$ & $\begin{array}{l}\text { Siewiorek et } \\
\text { al. (2012) }\end{array}$ \\
\hline
\end{tabular}




\begin{tabular}{|c|c|c|c|}
\hline & $\begin{array}{l}\text { descritivos e observação } \\
\text { das equipes. }\end{array}$ & $\begin{array}{l}\text { sua equipe; } \\
\text { - O ambiente de jogos de simulação tem o potencial de } \\
\text { uso no ensino superior para exercitar as habilidades de } \\
\text { liderança em contextos reais. }\end{array}$ & \\
\hline 25 & $\begin{array}{l}\text { - Simulação; } \\
\text { - Multi-método; } \\
\text { - Teste de hipóteses; } \\
\text { - Survey; } \\
\text { - Entrevista semi- } \\
\text { estruturada. }\end{array}$ & $\begin{array}{l}\text { - A aprendizado dos alunos foi aprimorado através do } \\
\text { uso de jogos de simulação como catalisador de } \\
\text { aprendizado e veículo de aprendizado; } \\
\text { - Os resultados contribuem para o debate acadêmico } \\
\text { sobre a implementação efetiva do treinamento baseado } \\
\text { em jogos de simulação em negócios e gestão } \\
\text { educacional. }\end{array}$ & $\begin{array}{l}\text { Loon, Evans e } \\
\text { Kerridge } \\
\text { (2015) }\end{array}$ \\
\hline 26 & $\begin{array}{l}\text { - Simulação de uma planta } \\
\text { virtual da cadeia de } \\
\text { suprimentos chamado } \\
\text { Logistic GameTM. }\end{array}$ & $\begin{array}{l}\text { As sessões de jogos analisadas mostram o poder } \\
\text { educacional e o valor do treinamento do Jogo Logístico } \\
\text { em Gestão de Operações; o jogo facilita a seleção de } \\
\text { empregos, conforme confirmado por todas as empresas } \\
\text { envolvidas. }\end{array}$ & $\begin{array}{l}\text { Battini et al. } \\
(2009)\end{array}$ \\
\hline 27 & $\begin{array}{l}\text { - Gamificação; } \\
\text { - Indústria de eletrônicos; } \\
\text { - Estudo de caso. }\end{array}$ & $\begin{array}{l}\text { Na prática, ampliando o entendimento e a aplicação do } \\
\text { pensamento enxuto através dos pressupostos da } \\
\text { gamificação, tem o potencial de motivar os funcionários } \\
\text { a se envolverem na organização e fornece aos gestores } \\
\text { ferramentas de controle. }\end{array}$ & $\begin{array}{l}\text { Pereira et al. } \\
(2018)\end{array}$ \\
\hline 28 & $\begin{array}{l}\text { - Desenvolvimento da } \\
\text { fábrica de aprendizado } \\
\text { Lean; } \\
\text { - Rastreamento contínuo } \\
\text { de pedidos de clientes } \\
\text { durante o } \\
\text { processo de fabricação } \\
\text { com base em dados em } \\
\text { tempo real. }\end{array}$ & $\begin{array}{l}\text { - Como resultado da simulação realizada em um } \\
\text { ambiente "Lean learning factory), foi possível ensinar a } \\
\text { aplicação adequada da Indústria } 4.0 \text { na produção } \\
\text { logística e práticas lean; } \\
\text { - Simulação ocorreu por meio de rodadas com diferentes } \\
\text { sistemas de produção; } \\
\text { - Foram analisados problemas específicos do } \\
\text { planejamento logístico no campo da "logística de } \\
\text { produção inteligente"; } \\
\text { - A pesquisa está em andamento. }\end{array}$ & $\begin{array}{l}\text { Blöchle } \\
\text { Schneider } \\
\text { (2016) }\end{array}$ \\
\hline 29 & $\begin{array}{l}\text { - Simular o ambiente de } \\
\text { uma auditoria em } \\
\text { funcionamento; } \\
\text { - Coorte. }\end{array}$ & $\begin{array}{l}\text { - A maioria dos estudantes consideraram a simulação útil } \\
\text { para a compreensão da teoria com a prática, mas não } \\
\text { houve envolvimento total com o material e atividades } \\
\text { (50\% dos casos). } \\
\text { - Comentários negativos dos participantes foram em } \\
\text { relação à instruções claras e alguns alunos não gostam } \\
\text { ou se recusam a se envolver com o ambiente de } \\
\text { aprendizado fora do padrão. }\end{array}$ & $\begin{array}{l}\text { Murdon e } \\
\text { Munro (2016) }\end{array}$ \\
\hline 30 & $\begin{array}{l}\text { - Beer Gam (jogo de } \\
\text { simulação); } \\
\text { - Uso de palestras e } \\
\text { sessões tutoriais; } \\
\text { - Estudo de caso. }\end{array}$ & $\begin{array}{l}\text { - A pesquisa apresenta um novo método de ensino e } \\
\text { avalia em um programa de cadeia de suprimentos para } \\
\text { torná-lo desafiador e interessante; } \\
\text { - Os resultados mostram que os alunos podem adquirir } \\
\text { ativamente o conhecimento da cadeia de suprimentos } \\
\text { por meio de simulação; } \\
\text { - Os alunos adotaram proativamente novas técnicas, } \\
\text { como sistemas de previsão colaborativa, inventário e } \\
\text { parceria estratégica com o fornecedor para melhorar o } \\
\text { desempenho da cadeia de suprimentos. }\end{array}$ & Lau (2015) \\
\hline 31 & - Jogo de simulação; & - O jogo de simulação facilitou o processo de & Otoiu e Otoiu \\
\hline
\end{tabular}




\begin{tabular}{|l|l|l|l|}
\hline - Teoria e prática sobre & aprendizado, no qual autonomia e responsabilidade & (2012) \\
Gestão de Recursos & foram vistas como duas grandes competências & facilitadoras e transferíveis. \\
Humanos; & - Para que o impacto do jogo seja positivo e relevante, & \\
- Duração de um & deve-se levar em consideração o fato de que o & \\
semestre; & aprendizado depende muito do envolvimento na tarefa. & \\
- Estudo de caso. & & \\
\hline
\end{tabular}

Fonte: Os autores (2019).

Conforme a análise dos trabalhos apresentados, destaca-se a importância do uso de jogos de simulação para educação e treinamento, ao que se deve o grau de similaridade entre o ambiente de treinamento a realidade industrial/empresarial. De forma geral, os estudos apontaram que o uso da simulação melhorou a aprendizagem e as habilidades dos participantes, que podem ser transferidas para empregos futuros.

Ainda, instrui os participantes à tomada de decisão, alcança os resultados de aprendizagem, promove o trabalho em equipe e desperta a motivação, aplica a teoria de maneira prática, tornando os alunos sujeitos ativos no processo de ensino-aprendizagem. No entanto, para iniciantes, os jogos de simulação não devem ser muito complexos uma vez que dificulta a compreensão dos participantes.

A Figura 5 apresenta os termos relevantes presentes nos resumos. Foram encontrados 664 termos e selecionadas as conexões (meet) mais relevantes (60\%). Em relação as palavraschave relevantes na pesquisa, de acordo com o Software VosViewer, destacam-se diferentes períodos e conexões. O tamanho de cada palavra na nuvem é proporcional à sua prevalência nos documentos selecionados (EL-ARINI; GUESTRIN, 2011). Foram identificados três clusters (vermelho, verde e azul), no qual as cores representam os termos das pesquisas que possuem vínculos e as linhas representam as ligações entre os artigos.

Figura 5 - Termos relevantes da pesquisa

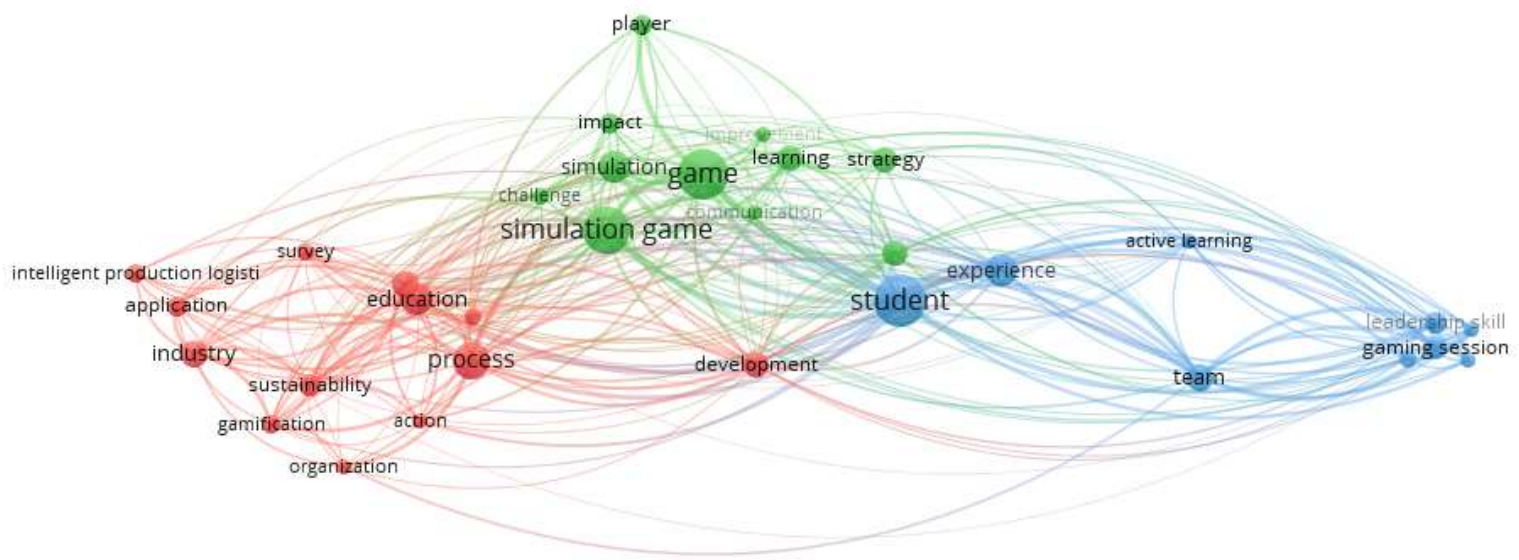

Fonte: Os autores (2019).

A análise da rede bibliográfica reflete a pesquisa realizada, em que os conteúdos estão 
relacionados entre si. No caso da contagem completa, as referências altamente citadas são vistas como mais representativas e não considera as referências pouco citadas; o que não é desejável. Portanto, usou-se da contagem fracionada em que cada referência é considerada igualmente representativa no PB (PERIANES-RODRIGUEZ; WALTMAN; VAN ECK, 2016). Os termos principais de pesquisa foram game, simulation game, challenge, education, process, development, student, active learning, team, gaming session, player, entre outros.

Por fim, conclui-se que a abordagem unificada do mapeamento bibliométrico permite um maior entendimento dos assuntos/termos atuais e suas relações no domínio de interesse.

\section{CONSIDERAÇÕES FINAIS}

Os estudos bibliométricos tornaram-se emergente, dada a importância e crescimento das produções científicas ao longo do tempo. Uma vez que os artigos científicos estão indexados em bases de dados, permitem identificar diferentes aspectos como palavras-chave, autores, citações, colaboração institucional, entre outras. Nesse sentido, a presente pesquisa realizou uma análise bibliométrica e análise de conteúdo, identificado a produção acadêmica, os autores, os periódicos e respectivo impacto (JCR), instituições e países, além da rede bibliométrica para o período de 10 anos (2009-2019).

Essa análise é relevante para conhecer a maturidade do assunto, importância que a comunidade acadêmica atribui, países e universidades que pesquisam. Considerando a atual demanda por metodologias ativas em sala de aula e sujeitos ativos no processo de ensinoaprendizagem, há muitas pesquisas que demonstram o uso de jogos e simulação como facilitador no processo de ensino.

Conforme pesquisa realizada, houve melhora no aprendizado se comparado ao ensino tradicional, especialmente para conteúdos considerados difíceis. Conclui-se que as várias formas interativas de uso dos jogos de simulação facilitam o processo de aprendizado, principalmente pelo maior envolvimento dos participantes, vivência de situações reais e práticas.

\section{REFERÊNCIAS}

BADURDEEN, F.; MARKSBERRY, P.; HALL, A.; GREGORY, B. Teaching lean manufacturing with simulations and games: A survey and future directions. Simulation \& Gaming, v. 41, n. 4, p. 465-486, 2010.

BARZILAI, S. "Half-reliable": A qualitative analysis of epistemic thinking in and about a digital game. Contemporary Educational Psychology, v. 51, p. 51-66, 2017.

BATTINI, D.; FACCIO, M.; PERSONA, A.; SGARBOSSA, F. Logistic Game ${ }^{T M}$ : learning by doing and 
knowledge-sharing. Production Planning \& Control, v. 20, n. 8, p. 724-736, 2009.

BHUSRY, Mamta; RANJAN, Jayanthi. Enhancing the teaching-learning process: a knowledge management approach. International Journal of Educational Management, v. 26, n. 3, p. 313329, 2012.

BLÖCHL, S. J.; SCHNEIDER, M. Simulation game for intelligent production logistics-The PuLL ${ }^{\circledR}$ learning factory. Procedia Cirp, v. 54, p. 130-135, 2016.

BUIL, I.; CATALÁN, S.; MARTÍNEZ, E. Encouraging intrinsic motivation in management training: The use of business simulation games. The International Journal of Management Education, $v$. 17, n. 2, p. 162-171, 2019.

BURDON, W.M.; MUNRO, K. Simulation-is it all worth it? The impact of simulation from the perspective of accounting students. The International Journal of Management Education, $v$. 15, n. 3, p. 429-448, 2017.

DAVIS, D.; CHEN, G.; HAUFF, C.; HOUBEN, G. J Activating learning at scale: A review of innovations in online learning strategies. Computers \& Education, v. 125, p. 327-344, 2018.

DE VIN, L.; JACOBSSON, L.; ODHE, J.E. Game-based lean production training of university students and industrial employees. Procedia Manufacturing, v. 25, p. 578-585, 2018.

EL-ARINI, K.; GUESTRIN, C. Beyond keyword search: discovering relevant scientific literature. In: Proceedings of the 17th ACM SIGKDD international conference on Knowledge discovery and data mining. ACM, 2011. p. 439-447.

GABRIELSSON, J.; TELL, J.; POLITIS, D. Business simulation exercises in small business management education: using principles and ideas from action learning. Action Learning: Research and Practice, v. 7, n. 1, p. 3-16, 2010.

GATTI, L.; ULRICH, M.; SEELE, P. Education for sustainable development through business simulation games: An exploratory study of sustainability gamification and its effects on students' learning outcomes. Journal of cleaner production, v. 207, p. 667-678, 2019.

GEITHNER, S.; MENZEL, D. Effectiveness of learning through experience and reflection in a project management simulation. Simulation \& Gaming, v. 47, n. 2, p. 228-256, 2016.

HAUGE, J.B.; RIEDEL, J.C. Evaluation of simulation games for teaching engineering and manufacturing. Procedia Computer Science, v. 15, p. 210-220, 2012.

HERNÁNDEZ-LARA, A.B.; PERERA-LLUNA, A.; SERRADELL-LÓPEZ, E. Applying learning analytics to students' interaction in business simulation games. The usefulness of learning analytics to know what students really learn. Computers in Human Behavior, v. 92, p. 600-612, 2019.

KIILI, K.; LAINEMA, T.; DE FREITAS, S.; ARNAB, S. Flow framework for analyzing the quality of educational games. Entertainment computing, v. 5, n. 4, p. 367-377, 2014. 
LAU, A.K.W. Teaching supply chain management using a modified beer game: an action learning approach. International Journal of Logistics Research and Applications, v. 18, n. 1, p. 62-81, 2015.

LI, Q.; JIANG, Y.; ZHANG, M. National representation in the emergency medicine literature: a bibliometric analysis of highly cited journals. The American journal of emergency medicine, $\mathrm{v}$. 30, n. 8, p. 1530-1534, 2012.

LIN, Hong-Wen; LIN, Yu-Ling. Digital educational game value hierarchy from a learners' perspective. Computers in Human Behavior, v. 30, p. 1-12, 2014.

LOON, M.; EVANS, J.; KERRIDGE, C. Reprint: Learning with a strategic management simulation game: A case study. The International Journal of Management Education, v. 13, n. 3, p. 371380, 2015.

OTOIU, C.; OTOIU, G. Testing a simulation game as a potential teaching method for a masters course in human resources management. Procedia-Social and Behavioral Sciences, v. 33, p. 845-849, 2012.

PASIN, F.; GIROUX, H. The impact of a simulation game on operations management education. Computers \& Education, v. 57, n. 1, p. 1240-1254, 2011.

PEREIRA, M.; OLIVEIRA, M.; VIEIRA, A.; LIMA, R. M.; PAES, L. The gamification as a tool to increase employee skills through interactives work instructions training. Procedia computer science, v. 138, p. 630-637, 2018.

PERIANES-RODRIGUEZ, A.; WALTMAN, L.; VAN ECK, N.J. Constructing bibliometric networks: A comparison between full and fractional counting. Journal of Informetrics, v. 10, n. 4, p. 11781195, 2016.

PETERKOVÁ, J.; WOZNIAKOVÁ, Z. The best practice in teaching process by using managerial simulation games. Procedia-Social and Behavioral Sciences, v. 174, p. 3862-3867, 2015.

RANCHHOD, A.; GURĂU, C.; LOUKIS, E.; TRIVEDI, R. Evaluating the educational effectiveness of simulation games: A value generation model. Information Sciences, v. 264, p. 75-90, 2014.

SCHWARZ, J.O. Business wargaming for teaching strategy making. Futures, v. 51, p. 59-66, 2013.

SEVERENGIZ, M.; ROEDER, I.; SCHINDLER, K.; SELIGER, G. Influence of Gaming Elements on Summative Assessment in Engineering Education for Sustainable Manufacturing. Procedia Manufacturing, v. 21, p. 429-437, 2018.

SHIH, J.L.; JHENG, S.C.; TSENG, J.J. A simulated learning environment of history games for enhancing players' cultural awareness. Interactive Learning Environments, v. 23, n. 2, p. 191211, 2015.

SIEWIOREK, A.; SAARINEN, E.; LAINEMA, T.; LEHTINEN, E. Learning leadership skills in a 
simulated business environment. Computers \& Education, v. 58, n. 1, p. 121-135, 2012.

SIEWIOREK, A.; SAARINEN, E.; LAINEMA, T.; LEHTINEN, E. Learning leadership skills in a simulated business environment. Computers \& Education, v. 58, n. 1, p. 121-135, 2012.

WEB OF KNOWLEDGE. Journal citation reports: information for new users. 2012. Available from: <http://admin-apps.webofknowledge.com/JCR/help/h_info.htm\#information>. Cited: 30 July 2012.

ZARZA, G.; LUGONES, D.; FRANCO, D.; LUQUE, E. An innovative teaching strategy to understand high-performance systems through performance evaluation. Procedia Computer Science, v. 9, p. 1733-1742, 2012. 